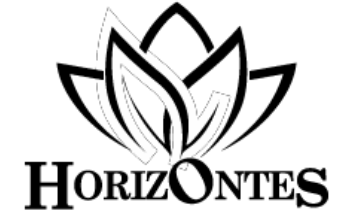

www.revistahorizontes.org
Horizontes. Revista de Investigación en Ciencias de la Educación Https://doi.org/10.33996/revistahorizontes.v2i6.49 abril - junio, 2018 Volumen 2 / No. 6 pp. $104-120$

\title{
Diseño Instruccional sobre equilibrio químico en fase gaseosa empleando Tecnologías de la Información y Comunicación en estudiantes de Química de la UPEL-IPC
}

\author{
Instructional Design of Chemical Equilibrium in the Gaseous Phase Using \\ Information and Communication Technologies in UPEL-IPC Chemistry \\ Students
}

\author{
Diana Sosa \\ Eury Castillo \\ dianamha2@gmail.com \\ yruecast@gmail.com \\ Universidad Pedagógica Experimental Libertador. Instituto Pedagógico de Caracas, Venezuela
}

\section{RESUMEN}

El presente estudio tuvo como propósito la elaboración de un diseño instruccional empleando Tecnología de Información y Comunicación (TIC) para optimizar los procesos de enseñanza y aprendizaje sobre el tópico equilibrio químico. Enmarcado en la modalidad de proyecto factible, de carácter cualitativo descriptivo y naturaleza emergente. Contemplándose en 4 fases. Los resultados de las entrevistas realizadas a los participantes arrojaron que dicha estrategia estuvo acorde a la necesidad real de los estudiantes, es decir, la estrategia tecnológica elaborada cumplió satisfactoriamente con las expectativas logrando principalmente reforzar el proceso de aprendizaje en los estudiantes. Se recomienda la aplicación a un mayor número de participantes y elaborar mayor cantidad de medios y recursos innovadores para el desarrollo de los tópicos de mayor dificultad en ciencias naturales.

Palabras clave: Tecnología de Información y Comunicación (TIC); diseño estratégico Instruccional emergente; equilibrio químico gaseoso; video educativo

\begin{abstract}
The purpose of this study was to develop an instructional design using Information and Communication Technology (ICT) to optimize the teaching and learning processes on the topical chemical balance. Framed in the feasible project modality, of descriptive qualitative nature and emerging nature. Contemplating in 4 phases. The results of the interviews with the participants that said strategy was in accordance with the students' real need, that is, the technological strategy elaborated satisfactorily met the expectations, mainly reinforcing the learning process in the students. It is recommended to apply to a greater number of participants and develop a greater amount of innovative means and resources for the development of the most difficult topics in natural sciences.
\end{abstract}

Key words: Information and Communication Technology (ICT); strategic design emergent instruction; gas chemical equilibrium; educational video 
INTRODUCCIÓN

La educación es un proceso que se encarga de socializar, concientizar y vincular bidireccionalmente la transferencia y enseñanza de los conocimientos, las aptitudes, actitudes, los valores, las potencialidades, costumbres, entre otros... a su vez, contribuye en la formación de personas virtuosas con valores fundamentales de vida, aspiraciones y autorrealización. El avance tecnológico está en constante actualización, da pasos agigantados en muchos ámbitos, y el campo educativo no se escapa de esta realidad y la evolución tecnológica va de la mano con el crecimiento educativo, requiriendo de innovación, herramientas, y alternativas para ir de la mano con la tecnología (Castillo, 2011).

La educación en Venezuela, está orientada siempre a mejorar el proceso educativo, bajo condiciones políticas que aseguren que todos los venezolanos reciban una educación gratuita y participativa, expresado así en el artículo $103^{\circ}$ de la Constitución de la República Bolivariana de Venezuela, "Toda persona tiene derecho a una educación integral, de calidad, permanente, en igualdad de condiciones y oportunidades", es inevitable que por lo menos un solo venezolano no reciba hoy día educación.

La metodología de la enseñanza responde a la manera de comprender la relación que se establece entre el sujeto que aprende y el objeto de conocimiento. Al respecto, Not (1987) nos habla de una confrontación clásica que se ha dado en el campo de la pedagogía entre los métodos de enseñanza. Por un lado, los métodos antiguos o tradicionales y por el otro los métodos modernos o activos.

Hoy en día es necesario el apoyo tecnológico para optimizar el proceso de Enseñanza-Aprendizaje, por lo que se ha convertido en una herramienta básica para el desarrollo de la misma. Cada avance en materia de ciencia y tecnología deja una huella en la calidad de vida de las personas que conforman la sociedad y por consiguiente:

Las TIC son un tipo de tecnología que nace a partir de los avances científicos producidos en los ámbitos de la informática y la comunicación. Las podemos definir como el conjunto de tecnologías que tienen como objetivo facilitar y mejorar la calidad de vida de las personas a través de las ventajas que nos proporcionan dichas tecnologías. La máxima expresión de las TIC la encontramos en Internet, lo que ha supuesto una revolución y ha redefinido la forma de relacionarse y comunicarse de las personas (Tuyú Tecnology, 2017).

Estas tecnologías se han vuelto tan indispensables que hasta para realizar la acción más simple y cotidiana (como, por ejemplo: movimientos de dinero, trámites bancarios, pago de servicios, comunicación con familiares y amigos, entre otros) y en consecuencia son utilizadas las TIC, de esta realidad no se escapa del ámbito educativo, laboral, y mucho menos del cotidiano, para todo contexto se requiere del manejo básico de la computadora, y del internet.

El ámbito educativo no se puede desligar de las tecnologías de información y comunicación. Es sabido que los jóvenes de hoy en día suelen hacer uso de las herramientas informáticas con suma facili-dad, y para todo, desde las redes sociales, hasta para la realización de sus actividades cotidianas y académicas (EDUCREA, 2018). 
Según Pérez (2010) "La realidad educativa ha dado un giro radical. Nuestros jóvenes traen incorporado el "chip" tecnológico y no es de sorprender que niños de edades tempranas se apropien de la tecnología", el autor explica claramente que hoy somos los adultos los que solicitamos ayuda para utilizar uno u otro aparato tecnológico. En la actualidad, los docentes dejaron de ser los poseedores del saber para convertirse en facilitadores del proceso educativo. Ahora es normal que el docente consulte a sus estudiantes o bien que el alumno comparta sus conocimientos tecnológicos con el docente.

En este entorno pedagógico, la función de las tecnologías de información y comunicación resulta fundamental. Las computadoras constituyen un valioso soporte en esta nueva tendencia, pero también se requieren pizarras inteligentes, notebookscomputadoras y sistemas para videoconferencias, entre otros.

Las Tecnologías de la Información y las Comunicaciones (TIC) han sido reconocidas como recursos innovadores que permiten diseñar un conjunto de estrategias en las prácticas docentes, capaces de producir una verdadera revolución educativa en general y en la enseñanza de las Ciencias Naturales en particular, en el marco de una revolución social que provocan estas tecnologías y que hoy en los ámbitos académicos ya no se discute (Kofman, 2005

Es por ello que hace esencial un método para que los estudiantes descubran, aprendan y logren competencias para desenvolverse en una sociedad que cambia constantemente y con exceso de información y así lograr elevar esa calidad del aprendizaje (EducarChile, 2008).

Una de las formas de optimizar los procesos de enseñanza $\mathrm{y}$ aprendizaje es mediante el diseño instruccional, el diseño instruccional se ocupa de la planeación, la preparación y el diseño de los recursos y ambientes necesarios para que se lleve a cabo el aprendizaje. Por otro lado, el diseño instruccional es la ciencia de creación de especificaciones detalladas para el desarrollo, implementación, evaluación, y mantenimiento de situaciones que facilitan el aprendizaje de pequeñas y grandes unidades de contenidos, en diferentes niveles de complejidad (Szczurek. 1990).

Ahora bien, el diseño instruccional representa hoy día la mejor forma de realizar los procesos de enseñanza aprendizaje, mediante diversos modelos que mejoran la educación en los jóvenes, elevando así la calidad educativa. Entre estos modelos se encuentra el propuesto por Szczurek (1989), el cual indica el camino a seguir para quienes deciden hacer desarrollo tecnológico en escenarios educativos.

Existe una problemática asociada a las ciencias naturales donde los diversos tópicos se tornan en ocasiones difíciles de enseñar, en pocas palabras, los recursos se hacen insuficientes repercutiendo así en los procesos de enseñanza-aprendizaje, lo expresa (Busquets, 2016). Por ello, resulta pertinente realizar el presente trabajo de investigación que tiene por objeto principal la realización de un diseño Instruccional empleando las TIC para optimizar los procesos de enseñanza y aprendizaje en los estudiantes de la especialidad de química del Instituto Pedagógico de Caracas.

Esta investigación tuvo como propósito el desarrollo de una alternativa innovadora empleando tecnología de información y la comunicación en un tópico de Química General.

En concordancia con la interrogante presentada y el propósito de la investigación fueron planteados los siguientes objetivos proponer una estrategia instruccional 
empleando Tecnología de Información y la Comunicación (TIC) para optimizar el proceso de enseñanza-aprendizaje en un tópico de Química General. Además de realizar un estudio de necesidades que permita conocer el tópico y la TIC emergente, para optimizar el proceso de enseñanza-aprendizaje de los estudiantes de Química General. Por otro lado, también se diseñó estrategia didáctica correspondiente al tópico emergente que permita optimizar el proceso de enseñanzaaprendizaje de los estudiantes de Química General. Fue validado por juicios de expertos la estrategia didáctica con enfoque TIC, sobre el tópico de Química General para optimizar el proceso de enseñanza-aprendizaje de los estudiantes de Química. Posteriormente, se implementó la estrategia didáctica que permitió optimizar el proceso de enseñanza y aprendizaje de los estudiantes de Química. Para finalizar se evaluó la estrategia instruccional correspondiente que permita optimizar el proceso de enseñanza y aprendizaje de los estudiantes de Química.

\section{MÉTODO}

Según Sabino (2002) la investigación se produce como un movimiento que enlaza la teoría y la práctica entre ambos se encuentra entonces el método y por sobre todo la metodología. La primera enfatiza el proceso de construcción y del cómo será conformado el conocimiento que se rige por determinadas pautas que le permiten al investigador obtener el carácter científico. El segundo movimiento la metodología está constituida por las orientaciones para hacer la tarea práctica por parte del investigador, por lo tanto, la recolección y organización de la información que se obtendrá en la realidad.

La investigación entonces puede ser de varios tipos, $\mathrm{y}$ en tal sentido se puede clasificar de distintas maneras, sin embargo, es común hacerlo en función de su nivel, su diseño y el propósito de la misma (Sabino, 2002).

Por lo tanto, la presente investigación fue realizada bajo la modalidad de Proyecto Factible debido a que se desarrolló una propuesta enfocada en una problemática educativa, apoyado en la investigación documental y de campo, contó el diseño de tipo emergente, por lo que la información surgió de manera sistemática a lo largo del estudio.

\section{Participantes de la investigación}

Fueron seleccionados de manera individual, es decir, no probabilística. De esta forma los individuos participantes no dependen de la probabilidad, sino que se ajustaron a las necesidades de la investigación (Hernández, Fernández y Baptista, 2006).

Los participantes estuvieron constituidos por un grupo reducido de estudiantes entre ellos hombres y mujeres de la especialidad de Química, cursantes de Química General, corte 2016-II, esto se debe a que actualmente no se dispone de material de impresión para hacer el estudio a una cantidad más elevada de estudiantes.

\section{Técnicas e Instrumentos de Recolec- ción de Datos}

La técnica que fue usada para la recolección de la información de datos del presente estudio es la entrevista, la define según Arias (2009) como "una técnica que consiste en visualizar o captar mediante la vista, en forma sistemática, cualquier hecho, fenómeno o situación que se produzca en la naturaleza o en la sociedad, en función de unos objetivos de investigación preestablecidos", haciendo especial referencia a la observación directa, ya que la indirecta se realiza a través de objetos muy sofisticados tales como: el microscopio, telescopio, entre otros. 
Entre tanto, es importante resaltar que la observación posee diversas categorías, entre todas las que presenta dicha técnica, la utilizada para esta investigación es la entrevista directa, como se había resaltado en líneas anteriores, puesto que el entrevistado tiene contacto directo con el entrevistador, en efecto, la observación participante, en este caso, el investigador pasó a ser parte de la comunidad o medio donde se desarrolló el estudio, siendo a su vez estructurada, cumpliendo con los objetivos preestablecidos en el estudio, utilizando una guía prediseñada, en la que se específica los ele-mentos observados.

Es importante resaltar en este aspecto que en la fase diagnóstica, a través de un instrumento realizado previamente para conocer el problema puntual que enfrentaban los estudiantes y posteriormente a manera de profundizar más se comienza entonces con la entrevista directa, participante y estructurada.

El Instrumento de papel y lápiz, el cual fue sometido a la validación por juicio de expertos, contempla una estructura mixta que incluye aspectos conceptuales, procedimentales y actitudinales estructurados en dos (2) ítems distribuidos en las 2 partes siguientes:

Parte A. la cual consiste en selección simple el encuestado señalará con una $\mathrm{X}$ uno de los tópicos del cual le gustaría que surja un material Instruccional de fuente tecnológica. Contemplado así como el primer ítem, el sub ítem A.1. El encuestado justificó brevemente porque se le dificultaba el tópico anteriormente seleccionado, si es para su aprendizaje o para la enseñanza en aula.

Parte B. La cual consistió también en la selección simple, marcando con una x la o las opciones acordes a su preferencia Contemplado así como el segundo ítem, el sub ítem B.1. Seleccionara que tipo de fuente tecnológica le parecerá la más apropiada para el posterior diseño Instruccional. Y el sub ítem B.2. Deberá ser llenado si el estudiando señaló en otro podrá entonces colocar la sugerencia del tipo de fuente tecnológica le parezca la más conveniente.

Para la medición de conducta se utilizó un cuestionario con preguntas cerradas, para medir la escala de actitud o de conducta, donde se establecieron una serie de instrucciones presentándose en afirmaciones de acuerdo a la escala de actitud anexada previamente.

\section{Fases o etapas del proyecto de investigación}

\section{Desarrollo Tecnológico Instruccional}

Szczurek (1990) define el desarrollo instruccional como el conjunto de supuestos y técnicas que deben tenerse en cuenta en los componentes del proceso de enseñanzaaprendizaje $y$ sus interrelaciones para ejecutar soluciones.

Por otro lado, Diez, (2001). Asegura que el desarrollo instruccional propuesto por los autores antes mencionados, ha servido como base para la elaboración de materiales educativos específicamente en el área de las Ciencias Naturales, en institutos tales como el CENAMEC, IVIC, INN, entre otros.

Ahora bien, el modelo de Desarrollo Instruccional, propuesto por estos autores, contempla 4 fases o etapas, (ver gráfico 1). Las cuáles serán presentadas a continuación: 


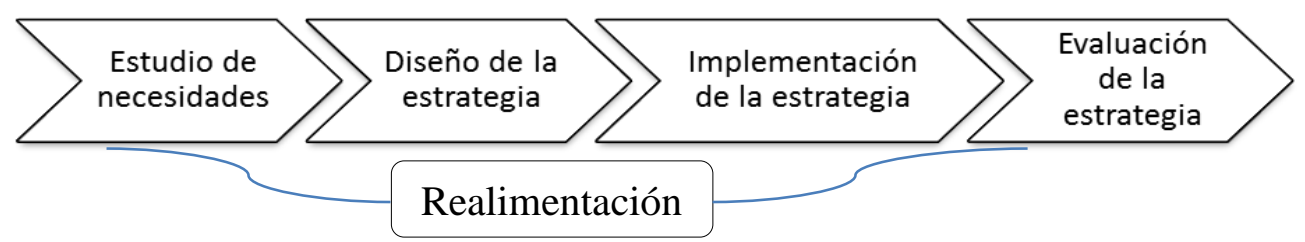

Gráfico 1. Modelo de Desarrollo Tecnológico Instruccional. Tomado de Szczurek (1990). Modelo Instruccional de maestría.

Para la ejecución de la primera fase del modelo de Desarrollo Tecnológico Instruccional (Muller y Szczurek, 1989), se siguió el modelo propuesto por Muller (1989), el cual comprende los siguientes pasos:

Detección de necesidades (Fase Diagnóstica). Es la etapa inicial de contacto con la realidad y con el deber ser. En esta etapa el objetivo es descubrir dónde estamos, de dónde partimos, qué es lo deseable, cómo obtener la información que necesitamos para determinar las discrepancias entre lo que está sucediendo y lo que debería suceder. Se identifican, documentan y justifican discrepancias en relación con insumos, procesos y resultados finales, utilizando un enfoque sistémico. Comprende los siguientes pasos:

- Identificar la situación actual: en este proceso, tenemos que ir a la realidad y determinar los posibles problemas que presenta la población afectada en cuanto al tema planteado. Por ello, Aquí se encuentran diversas y/o carencias que pueden dar una idea sobre la posibilidad de comenzar un Estudio de Necesidades que permita diagnosticar el problema que presenta el proceso de Enseñanzaaprendizaje en uno de los temas de Química General, por la cual una de las soluciones que se plantea también en el presente trabajo de investigación, es el de implementación de las Tecnologías de la Información y Comunicación que mejore hasta en el mejor de los casos, optimice dicho proceso para así mejorar la calidad educativa en los estudiantes de Química a futuros docentes del área.

- Identificar la situación deseable: se busca lo que debería ser, respondiendo a la pregunta ¿Dónde deberíamos estar? Se emplea una formulación en términos de los hechos que deberían estar sucediendo, de acuerdo a los establecido (dónde o por quién). Se identifican los niveles de desempeño requeridos bien sean normados, sentidos o deseados.

Análisis de necesidades. Esta etapa responde a la búsqueda del tipo de información que se requiere para comprender mejor el sistema en estudio y para poder enunciar las necesidades con precisión. La información que se requiere en esta fase, en términos de juicios y resultados que llevaran a jerarquizar y racionalizar las necesidades una vez captadas.

Proposición de solución. Ya identificado el problema se tiene que proponer la solución, es decir, se propone la estrategia didáctica con enfoque TIC a la problemática surgida en la primera fase, la cual pueda ser cualquier tema o tópico referente a Química General en que los estudiantes a futuro docente tiendan a tener dificultad para su proceso de Enseñanza-aprendizaje. 
Diseño de la estrategia didáctica correspondiente al tópico que surja en la problemática y que permita así optimizar el proceso de enseñanza-aprendizaje de los estudiantes de Química General.

Validación por juicios de expertos, se procederá con la validación de la estrategia didáctica empleando las TIC, por tres profesores de la especialidad de Química, sobre el tópico de Química General para optimizar el proceso de enseñanzaaprendizaje de los estudiantes de Química General.

\section{Implementación de la estrategia didáctica} al grupo de participantes o en este caso, los informantes del curso de Química General corte 2016-2.

Evaluación de la estrategia instruccional la cual surja y que permita así optimizar el proceso de enseñanza y aprendizaje de los estudiantes de Química.

Divulgación y Defensa del Trabajo de investigación para el transcurso del semestre.

\section{La categorización}

En investigación cualitativa, la categorización se constituye en una parte fundamental para el análisis e interpretación de los resultados "Este proceso consiste en la identificación de regularidades, de temas sobresalientes, de eventos recurrentes y de patrones de ideas en los datos provenientes de los lugares, los eventos o las personas seleccionadas para un estudio. La categorización constituye un mecanismo esencial en la reducción de la información recolectada". (Strauss y Corbin, 2002)

Las categorías son las diferentes valores, alternativas es la forma de clasificar conceptuar o codificar un término o expresión de forma clara que no se preste para confusiones a los fines de determinada investigación. En dichas alternativas serán ubicados, clasificados, cada uno de los elementos sujetos a estudio (las unidades de análisis).

El mismo autor señala lo siguiente:
La categorización consiste en la
asignación de conceptos a un nivel
abstracto... las categorías tienen un
poder conceptual puesto que
tienen la capacidad de reunir
grupos de conceptos o sub-
categorías. En el momento en el
que el investigador empieza a
agrupar los conceptos, también
inicia el proceso de establecer
posibles relaciones entre conceptos
sobre el mismo fenómeno. (Strauss
y Corbin, op cit)

Por lo que, las categorías son conceptos derivados de los datos que representan fenómenos, los fenómenos son ideas analíticas pertinentes que emergen de datos.

Si se plantea el fenómeno como hecho observable en un contexto específico del que se extracta una serie de información, es válido establecer un entramado de datos que se registran, a su vez esta información se convierte en campos definidos de análisis los que por sus características similares se organizan en categorías.

\section{Tratamiento de los Datos}

De acuerdo al tipo y diseño de investigación se llevó a cabo un análisis descriptivo y cualitativo, una vez que comenzó la recolección de los datos mediante la entrevista directa, participativa y estructurada, la solución que se propuso emergió de los datos obtenidos a lo largo del presente estudio. 
El procedimiento de muestreo donde fue dirigido por la lógica y es el propósito de los tres tipos básicos de procedimientos de codificación Strauss y Corbin (2002), siendo estos: la codificación abierta, la axial y la selectiva. Donde la codificación abierta fue la utilizada en el presente trabajo de investigación, el mismo autor describe dicha codificación como, "el proceso analítico por medio del cual se identifican los conceptos y se descubren en los datos sus propiedades y dimensiones".

Entonces, para descubrir, nombrar y desarrollar los conceptos se debe abrir el texto y exponer los pensamientos, ideas y significados contenidos en él. Sin este primer paso analítico, no puedo darse el resto del análisis y la comunicación subsiguiente. Hablando en términos generales, durante la codificación abierta, los datos se descomponen en partes discretas, se examinan minuciosamente y se comparan en busca de similitudes y diferencias. Los acontecimientos, sucesos, objetos y acciones o interacciones que se consideran conceptualmente similares en su naturaleza o relacionados en el significado se agrupan bajo conceptos más abstractos, denominados "categorías". El examen minucioso de los datos para encontrar diferencias y similitudes permite una sutil discriminación y una diferenciación entre categorías. (Strauss y Corbin, 2002).

\section{RESULTADOS}

A lo largo de la investigación se obtuvieron una serie de resultados en cada una de las fases que se detallan a continuación:

Diagnóstica: (a) conociendo la necesidad actual de los estudiantes de Química General por medio de un pre test, indicando en él, el tópico o tema que le sea dificultoso y la estrategia Instruccional con enfoque tecnológico que le gustaría surgiera.

Diseño: (b) del recurso tecnológico que emergió como video educativo estructurado en atención a la necesidad del tema que para entonces los estudiantes estaban teniendo necesidad incluyendo la validación e implementación de la misma.

Implementación (c) ejecución de la solución instruccional seleccionada durante el semestre.

Evaluación (d) de la estrategia didáctica tecnológico emergida y de los resultados más relevantes obtenidos.

\section{Validación por juicio de expertos del instrumento diagnóstico}

Una vez diseñada la prueba diagnóstica se procedió a su validación por juicio de expertos. El cuadro 1 resume las observaciones y aportes de los expertos durante el proceso de validación. 
Cuadro 1. Registro de observaciones en el proceso de validación del instrumento diagnóstico por juicio de expertos

\begin{tabular}{cl}
\hline Experto & \multicolumn{1}{c}{ Aporte } \\
\hline 1 & Recomendó mejorar la redacción en cada ítem y también consideró agregar un \\
& ítem que estuviera dirigido si la dificultad es para su aprendizaje o enseñanza \\
2 & Recomendó mejorar la redacción en cada ítem \\
3 & Consideró que el instrumento diagnóstico estaba bien, sin embargo comenta que se \\
& debía aclarar un poco la redacción de cada ítem.
\end{tabular}

Las observaciones recogidas en el cuadro 2 se tomaron en consideración en su totalidad durante el desarrollo de la misma, la experiencia de los expertos juega un papel importante ya que una vez que se realizaron dichos ajustes a dicho instrumento se pudo ejecutar satisfactoriamente el instrumento con el fin de diagnosticar cual es la problemática que estaban atravesando los participantes en estudio.

\section{Resultados del Estudio de Necesidades}

Partiendo de la primera fase siendo esta del estudio de necesidades, siendo aplicada a una pequeña población tomados como informantes en estudio, de ambos sexos, pertenecientes a la especialidad de Química en su totalidad, del segundo semestre corte 2016-II del Instituto Pedagógico de Caracas.

Por lo tanto, los resultados obtenidos a través del estudio de necesidades aplicados a dicha población, se presenta en el siguiente cuadro.

Cuadro 2. Selección del tópico de Química General y de la estrategia didáctica con enfoque TIC realizada por los participantes en estudio.

\begin{tabular}{llll}
\hline \multicolumn{1}{c}{ Tópico } & $\begin{array}{c}\text { Tópico } \\
\text { seleccionado }\end{array}$ & \multicolumn{1}{c}{ Estrategia /TIC } & $\begin{array}{c}\text { Estrategia /TIC } \\
\text { seleccionada }\end{array}$ \\
\hline Teoría atómica & & Blog & \\
Disoluciones & & Video educativo & \\
Propiedades físicas y & & Módulo Instruccional & \\
químicas de la materia & & Página web & \\
Nomenclatura & & Material instruccional & Video educativo \\
Estequiometria & Equilibrio & digital & \\
& químico & Aula virtual & \\
Reacciones químicas & & Prueba digital en & \\
Gases & & formulario de google & \\
& & Otro & \\
Equilibrio químico & & \\
Estructura atómica & & & \\
Electroquímica & & & \\
Enlace químico & & & \\
Tabla periódica & & & \\
\hline
\end{tabular}


Como se puede observar en el cuadro 2, el resultado más concluyente del estudio diagnóstico fueron que para el tópico de Química General donde los estudiantes presentaban dificultad para su aprendizaje es en Equilibrio químico, la estrategia seleccionada por los participantes en estudio fue la de Video educativo.

Una vez diagnosticada propiamente la necesidad de los participantes en estudio, la población que conforman el curso de Química General, los datos fueron procesados inmediatamente, por lo cual, se procede a la aplicación de una entrevista directa, participativa estructurada con el fin de conocer a profundidad y con más detalle del por qué la selección del tema de Equilibrio Químico y que parte específicamente de él tuvieron la necesidad de seleccionar el video educativo como estrategia didáctica tecnológica para la solución de la necesidad en evidencia, entretanto, el instrumento utilizado posteriormente para esta entrevista fue la grabadora.

A continuación se muestra en el cuadro 3, la conceptualización codificada abiertamente en la primera entrevista.

Cuadro 3. Entrevista realizada a los participantes en estudio con la finalidad de conocer la conceptualización de la fase diagnóstica.

\begin{tabular}{|c|c|c|c|c|}
\hline \multirow[t]{2}{*}{ Entrevistador } & \multicolumn{3}{|c|}{ Participantes } & \multirow[b]{2}{*}{ Conceptualización } \\
\hline & $\mathbf{P}_{1}$ & $\mathbf{P}_{2}$ & $\mathbf{P}_{3}$ & \\
\hline $\begin{array}{c}\text { 1. ¿Por qué se te } \\
\text { hace difícil el tema } \\
\text { de Equilibrio } \\
\text { Químico y que } \\
\text { parte } \\
\text { específicamente } \\
\text { de este tema } \\
\text { deseas solucionar? }\end{array}$ & $\begin{array}{l}\text { El que más } \\
\text { trabajé fue el } \\
\text { Equilibrio } \\
\text { gaseoso si lo } \\
\text { comprendí un } \\
\text { poco, sin } \\
\text { embargo, en } \\
\text { cuanto al } \\
\text { análisis de Kc } \\
\text { se me hizo } \\
\text { dificultoso por } \\
\text { las } \\
\text { comparacione } \\
\text { s que había que } \\
\text { hacer. }\end{array}$ & $\begin{array}{l}\text { Considero que } \\
\text { en la parte de } \\
\text { la constante } \\
\text { de equilibrio } \\
\text { Kc fue un poco } \\
\text { complicado al } \\
\text { trabajarlo } \\
\text { cuando hay } \\
\text { que aplicar el } \\
\text { análisis o la } \\
\text { respuestas a } \\
\text { los ejercicios }\end{array}$ & $\begin{array}{l}\text { Se me hace } \\
\text { difícil el } \\
\text { equilibrio } \\
\text { más que } \\
\text { todo } \\
\text { gaseoso al } \\
\text { sacar la } \\
\text { constante } \\
\text { de } \\
\text { equilibrio y } \\
\text { su análisis } \\
\text { cuando la } \\
\text { expreso. }\end{array}$ & $\begin{array}{l}\mathrm{P}_{1 .-} \text { Tema dificultoso, } \\
\text { Argumentar el análisis } \\
\text { de la constante, } \\
\text { igualación de la } \\
\text { constante ante otra. } \\
\mathrm{P}_{2 .-} \text { Constante de } \\
\text { equilibrio, Argumento de } \\
\text { los ejercicios, } \\
\text { razonamiento lógico. } \\
\mathrm{P}_{3} \text { - Expresar } \\
\text { numéricamente }\end{array}$ \\
\hline $\begin{array}{l}\text { 2. Como recurso } \\
\text { señalaste video } \\
\text { educativo, ¿Qué te } \\
\text { gustaría ver en el } \\
\text { video que hiciera } \\
\text { más referencia en } \\
\text { cuanto al tema? }\end{array}$ & $\begin{array}{l}\text { El análisis } \\
\text { escrito me } \\
\text { hacía ruido, } \\
\text { pienso que el } \\
\text { video es una } \\
\text { forma de } \\
\text { entender esa } \\
\text { parte más si lo } \\
\text { comparan } \\
\text { entre ellos con } \\
\text { la Qc, porque } \\
\text { yo soy más } \\
\text { visual que de } \\
\text { audio. }\end{array}$ & $\begin{array}{l}\text { Considero que } \\
\text { puede } \\
\text { trabajarse con } \\
\text { un video } \\
\text { educativo, } \\
\text { bueno por lo } \\
\text { menos en mi } \\
\text { caso pienso } \\
\text { que soy más } \\
\text { visual para } \\
\text { mi } \\
\text { aprendizaje y } \\
\text { hacer } \\
\text { hincapié en el }\end{array}$ & $\begin{array}{l}\text { Elegí la } \\
\text { parte de } \\
\text { video ya } \\
\text { que se me } \\
\text { hace más } \\
\text { fácil para } \\
\text { mi } \\
\text { comprensi } \\
\text { ón por sus } \\
\text { imágenes } \\
\text { para } \\
\text { visualizar } \\
\text { las cosas y } \\
\text { el audio que }\end{array}$ & $\begin{array}{l}\mathrm{P}_{1 .}-\text { Proceso de } \\
\text { aprendizaje, semejanzas } \\
\text { ante otra constante, } \\
\text { cognición de } \\
\text { aprendizaje. } \\
\mathrm{P}_{2 .}-\text { Estrategia } \\
\text { Instruccional } \\
\text { tecnológica, proceso de } \\
\text { aprendizaje, resaltar } \\
\text { hacer inferencias, } \\
\text { razonamiento lógico del } \\
\text { ejercicio matemático. } \\
\mathrm{P}_{3 .}-\text { Ilustraciones, } \\
\text { estrategias prácticas, }\end{array}$ \\
\hline
\end{tabular}




\begin{tabular}{|c|c|c|c|c|}
\hline \multirow[t]{3}{*}{ Entrevistador } & \multicolumn{3}{|c|}{ Participantes } & \multirow[b]{2}{*}{ Conceptualización } \\
\hline & $\mathbf{P}_{1}$ & $\mathbf{P}_{2}$ & $\mathbf{P}_{3}$ & \\
\hline & & $\begin{array}{l}\text { video en } \\
\text { cuanto a los } \\
\text { gases a la } \\
\text { interpretació } \\
\text { n y } \\
\text { comparación } \\
\text { de Kc y Qc } \\
\text { más allá que } \\
\text { numérica. }\end{array}$ & $\begin{array}{l}\text { lo puedo } \\
\text { reproducir } \\
\text { cuando yo } \\
\text { quiera, por } \\
\text { medio de } \\
\text { las } \\
\text { imágenes } \\
\text { me puedo } \\
\text { guiar por } \\
\text { las } \\
\text { reacciones, } \\
\text { la } \\
\text { constante } \\
\text { de } \\
\text { equilibrio } \\
\text { me cuesta } \\
\text { un poco } \\
\text { entenderla } \\
\text { en mi caso. }\end{array}$ & $\begin{array}{l}\text { ejemplificación con } \\
\text { imágenes, proceso de } \\
\text { aprendizaje significativo. }\end{array}$ \\
\hline
\end{tabular}

En el cuadro 4 se puede observar la entrevista realizada previamente a los participantes en estudio con el único fin de aclarar el por qué señalaron dicho tópico y cual parte de ese tema se les hacía dificultoso de entender, para ello, se hizo apropiado el uso de la conceptualización, según lo señala Strauss y Corbin (2002), Un concepto es un fenómeno al que se le ha puesto una etiqueta. Se trata de una representación abstracta de un acontecimiento, objeto o acción/ interacción que un investigador identifica como significativo en los datos. El propósito de denominar a los fenómenos es permitir a los investigadores reunir acontecimientos, sucesos $\mathrm{u}$ objetos similares bajo un encabezamiento clasificativo común. Aunque los acontecimientos o sucesos puedan ser elementos discretos, el hecho de que compartan características comunes y signi-ficados relacionados permite agruparlos. Con el fin de agrupar entonces los principales fenómenos referentes al tema de equilibrio químico, y de la estrategia más funcional para solventar la situación, en el hecho de la conceptualización bajo la codificación se pueden resaltar dicho fenómeno el cual es Equilibrio Químico en fase gaseosa siendo más que todo el análisis de Kc y Qc haciendo énfasis todos en que la parte de razonamiento de la constante de equilibrio, se puede evidenciar que no tienen una idea clara de lo que se pretende aclarar posteriormente al hacer los cálculos de dichas constantes, por lo tanto, los participante sugieren que la elaboración de un video educativo que haga especial énfasis en dicho fenómeno sería la mejor opción para solventar dicha situación, por ello, el presente trabajo de investigación toma el rumbo de realizar un video educativo como estrategia Instruccional enfocado a la utilización de las Tecnologías de Información y la Comunicación surgido entonces a través del estudio realizado.

\section{Estrategias instruccionales}

Mediante la aplicación del estudio diagnóstico, como vimos en párrafos anteriores, los estudiantes pertenecientes al 
curso de Química General, optaron entre todas las estrategias tecnológicas propuestas para el tópico que ellos también escogieron, la elaboración de un Video Educativo, el cual, es una herramienta didáctica sencilla y muy útil para el reforzamiento de los aprendizajes dentro y fuera del aula, ya que unas de las tantas características positivas del uso de esta estrategia es que pueden estar disponibles en la Internet, siendo más específicos, YouTube. Gardié, (1995) señala en su trabajo titulado: modelos de enseñanza creativa para la formación y el desempeño del docente venezolano, que es indispensable el uso de estrategias didácticas innovadoras (herramientas virtuales, videos educativos o instruccionales, laboratorios digitales, estrategias con enfoque contextualizado o CTS, simulaciones-juegos) que brinden al estudiante la oportunidad de potenciar el uso integral del cerebro en el desarrollo de habilidades cognitivas en el proceso de aprendizaje. En tal sentido, el uso de los recursos tecnológicos didácticos y programados por el docente que sean propios de los gustos y los estilos de aprendizaje de los estudiantes involucrados en el hecho destacándose así el video educativo como se señaló anteriormente, emergiendo entonces de la necesidad de los alumnos.

\section{Diseño del Video Educativo}

El diseño de la producción del video educativo, al tratarse de una realización audiovisual de naturaleza aplicada (en este caso al ámbito de la enseñanza y aprendizaje), debe tener una fase muy importante que es la de escribir el guion, según lo señala Vergmann y Sams, (2016), se debe tratar de convertir el contenido académico en un relato audiovisual utilizando estrategias y técnicas narrativas. Es la base fundamental del proceso, de la que depende en gran medida el resultado del proyecto audiovisual, en cuanto a la consecución de los objetivos enunciados en el guion.

El formato utilizado para la elaboración del Guion Técnico utilizado para la realización del video educativo, está bajo los formatos elaborados por el Departamento de Tecnología Educativa, a continuación observar el cuadro 4 y 5 referente a los guiones

Cuadro 4. Guion técnico utilizado para la elaboración del video educativo. Formato de cuadro del Departamento de Tecnología Educativa. (Adaptado al manual de la UPEL)

\begin{tabular}{lll}
\hline \multirow{2}{*}{ VIDEO 1 } & Para: Fase De Ejecución De Proyecto Educativo & Producción: \\
& Tema: Equilibrio químico (parte I) & Diana Sosa \\
& Sugerencia: Ninguna & Duración: 8 min. \\
\hline
\end{tabular}

\section{Presentación del recurso didáctico.}

Contenido a tratar.

Texto: Sistemas abiertos y cerrados ejemplos.

Fundamentación de equilibrio, equilibrio químico, características del equilibrio químico.

Explicación de un mapa conceptual de equilibrio químico, enfocándonos solamente en el equilibrio homogéneo en una sola fase.

Escritura de la constante de equilibrio.

¿Qué nos indica la constante de equilibrio?

Despedida: se invita a ver la segunda entrega de equilibrio químico donde se hablará de los factores que la afectan. 
Cuadro 5. Guion técnico utilizado para la elaboración del video educativo 2 da parte. Formato de cuadro del Departamento de Tecnología Educativa. (Adaptado a las normas UPEL)
Para: Fase De Ejecución De Proyecto Educativo
Tema: Equilibrio químico (parte II)

VIDEO 2

Sugerencia: ver el video 1
Producción:

Diana Sosa

\section{Presentación del recurso didáctico.}

Duración: $10 \mathrm{~min}$.

Contenido a tratar.

Texto: factores que afectan a la constante de equilibrio, variaciones de la concentración, presión, temperatura explicación del principio de Le chatelier.

Escritura de la constante de equilibrio.

Ejercicios de equilibrio químico (sugerencias de toma de notas al espectador). Ejercicio y razonamiento de la constante de equilibrio.

Predicción de la dirección de la constante de equilibrio. Ejercicio, solución y razonamiento.

Aplicaciones en la ciencia, tecnología y sociedad de equilibrio químico CTS.

Despedida y agradecimientos al espectador.

Se debe aclarar, que toda producción audio-visual debe tener su respectivo Guion técnico, puesto que es considerado un prerequisito para dar el siguiente paso que es el de la elaboración del video y más aún si este es con fines pedagógicos.
Una vez diseñada la unidad didáctica se procedió a su validación por juicio de expertos. El cuadro 6 resume las observaciones y aportes de los expertos durante el proceso de validación.

Cuadro 6. Registro de observaciones en el proceso de validación del Diseño Instruccional por juicio de expertos.

\begin{tabular}{cl}
\hline Experto & \multicolumn{1}{c}{ Aporte } \\
\hline 1 & $\begin{array}{l}\text { Recomendó aplicar música al inicio del video y sugiere agregar un video extra a } \\
\text { manera de práctica como apoyo externo. }\end{array}$ \\
2 & $\begin{array}{l}\text { Consideró que se debe aplicar más animaciones en la transición de la interfaz en el } \\
\text { video. }\end{array}$ \\
3 & Sugirió aumentar el tono de voz en el video \\
\hline
\end{tabular}

Las observaciones recogidas en el cuadro 6 deben ser consideradas en su totalidad al momento del diseño del video educativo. La experiencia de los expertos juega un papel importante ya que una vez que se realicen dichos ajustes al video educativo se puede evidenciar un cambio más beneficioso de los estudiantes.

\section{La aplicación del diseño instruccional}

Una vez elaborado el video educativo y pasado por una serie de estándares de validación, se aplicó el diseño, a través de la técnica de la entrevista directa, participativa y estructurada donde se utilizaron como instrumento la grabadora y la encuesta para medir la escala de actitud de los participantes posteriormente de a ver visto el diseño final, los resultados se pueden observar en el cuadro 7 , mostrándose a continuación. 
Cuadro 7. Implementación de la Estrategia Instruccional Tecnológica, entrevista realizada a los participantes en estudio.

\begin{tabular}{|c|c|c|c|c|}
\hline \multirow[b]{2}{*}{ Entrevistador } & \multicolumn{3}{|c|}{ Participantes } & \multirow[b]{2}{*}{ Conceptualización } \\
\hline & $\mathbf{P}_{1}$ & $P_{2}$ & $\mathbf{P}_{3}$ & \\
\hline $\begin{array}{l}\text { 1. El contenido } \\
\text { tratado, } \\
\text { ¿Cumplió con } \\
\text { las expectativas } \\
\text { que esperabas } \\
\text { conseguir? }\end{array}$ & $\begin{array}{l}\text { Sí, porque la } \\
\text { idea era dar } \\
\text { una } \\
\text { instroducci } \\
\text { ón general } \\
\text { en cuanto al } \\
\text { análisis de } \\
\text { Kc y Qc. Fue } \\
\text { pertinente } \\
\text { el contenido } \\
\text { con la } \\
\text { situación } \\
\text { actual. }\end{array}$ & $\begin{array}{l}\text { Sí, describe } \\
\text { lógicamente } \\
\text { paso por paso } \\
\text { los ejercicios } \\
\text { permitió } \\
\text { entender o } \\
\text { aclarar la } \\
\text { duda que se } \\
\text { traía, es decir, } \\
\text { permitió } \\
\text { hacer un } \\
\text { aprendizaje } \\
\text { significativo } \\
\text { de este } \\
\text { contenido. }\end{array}$ & $\begin{array}{l}\text { Sí, ya que pude } \\
\text { solventar mi } \\
\text { duda en } \\
\text { cuanto a las } \\
\text { comparacion } \\
\text { es de ambas } \\
\text { constantes, el } \\
\text { razonamient } \\
\text { o en los } \\
\text { ejercicios fue } \\
\text { claro y lógico, } \\
\text { ahora sí puedo } \\
\text { saber cómo } \\
\text { argumentar } \\
\text { un análisis en } \\
\text { estas } \\
\text { expresiones } \\
\text { matemáticas. }\end{array}$ & $\begin{array}{l}\mathrm{P}_{1 .-} \text { Fundamentos básicos, } \\
\text { objetivo de acuerdo con la } \\
\text { realidad. } \\
\mathrm{P}_{2 .} \text {-Pasos racionales, } \\
\text { soluciona problemas, } \\
\text { cumple con las teorías de } \\
\text { aprendizaje. } \\
\mathrm{P}_{3 .}-\text { Solucionó dudas, hizo } \\
\text { comparaciones } \\
\text { matemáticas, aclara como } \\
\text { se debe analizar un } \\
\text { ejercicio matemático. }\end{array}$ \\
\hline $\begin{array}{l}\text { 2. En cuanto a } \\
\text { la explicación, } \\
\text { ¿Cómo te } \\
\text { pareció la } \\
\text { narración } \\
\text { explicativa? }\end{array}$ & $\begin{array}{l}\text { Me parece } \\
\text { que estuvo } \\
\text { muy bien, } \\
\text { ya que puedo } \\
\text { entender } \\
\text { bien los } \\
\text { ejercicios } \\
\text { propuestos } \\
\text { en el video, } \\
\text { se veían } \\
\text { bastante } \\
\text { fáciles. }\end{array}$ & $\begin{array}{l}\text { Si fue clara } \\
\text { precisa y } \\
\text { concisa, los } \\
\text { entendí. }\end{array}$ & $\begin{array}{l}\text { La teoría } \\
\text { estuvo } \\
\text { bastante clara, } \\
\text { los ejercicios } \\
\text { estuvieron lo } \\
\text { bastante } \\
\text { explicativos. }\end{array}$ & $\begin{array}{l}\mathrm{P}_{1 .-} \text { Aceptación parcial. } \\
\mathrm{P}_{2 .-} \text { Aceptación positiva. } \\
\mathrm{P}_{3 .} \text { Afirmaciones } \\
\text { positivas }\end{array}$ \\
\hline $\begin{array}{l}\text { Recomiendas } \\
\text { mejorar algo en } \\
\text { el video, ya sea } \\
\text { en cuanto al } \\
\text { contenido, } \\
\text { diseño o en la } \\
\text { explicación. }\end{array}$ & $\begin{array}{l}\text { Si } \\
\text { recomiendo } \\
\text { mejorar } \\
\text { algo del } \\
\text { sonido, } \\
\text { ponerle } \\
\text { música por } \\
\text { lo menos al } \\
\text { inicio. }\end{array}$ & $\begin{array}{l}\text { No } \\
\text { recomiendo } \\
\text { mejorar nada } \\
\text { porque me } \\
\text { pareció } \\
\text { bonito y } \\
\text { atractivo el } \\
\text { diseño, no } \\
\text { está cargado } \\
\text { de contenido. }\end{array}$ & $\begin{array}{l}\text { Le podrías } \\
\text { mejorar } \\
\text { poniéndole } \\
\text { algo de } \\
\text { música al } \\
\text { comienzo y } \\
\text { que en los } \\
\text { ejercicios y la } \\
\text { parte CTS } \\
\text { aparezcan } \\
\text { uno por uno y } \\
\text { no al mismo } \\
\text { tiempo, de } \\
\text { resto todo } \\
\text { bien. }\end{array}$ & $\begin{array}{l}\mathrm{P}_{1 .-} \text { Aprendizaje auditivo. } \\
\mathrm{P}_{2 .-} \text { Aprendizaje audio- } \\
\text { visual abstracto } \\
\mathrm{P}_{3 .-} \text { Aprendizaje } \\
\text { audiovisual. }\end{array}$ \\
\hline
\end{tabular}


Ahora bien, en la primera pregunta realizada a cada uno de los participantes, para el Participante $1\left(\mathrm{P}_{1}\right)$, los conceptos surgidos son los siguientes: Fundamentos básicos, objetivo de acuerdo con la realidad, como se evidencia en el cuadro anterior, resultando ser positivo puesto que dice que contiene todos los fundamentos teóricos del tema de equilibrio químico en la fase gaseosa y está de acuerdo con que el video se apega a su realidad actual.

Seguidamente, para el Participante 2 $\left(\mathrm{P}_{2}\right)$, los conceptos surgidos son: Pasos racionales, soluciona problemas, cumple con las teorías de aprendizaje, al mencionar que en el video se describe lógicamente y paso por paso los ejercicios, quiere decir que el video le ayudó a mejorar su problema de la realización prácticas de ejercicios puesto que resaltó que le dejó aprendizajes significativos, es decir, según las teorías ausbelianas, piategeanas entre otras, se cumplen eficazmente en este aspecto.

Luego, para el Participante $3\left(\mathrm{P}_{3}\right)$, los conceptos surgidos son: Solucionó dudas, hizo comparaciones matemáticas, aclara como se debe analizar un ejercicio matemático. Podemos ver que a primera entrada afirma totalmente que solucionó sus dudas y que las comparaciones matemáticas le ayudaron aún más para su proceso de aprendizaje, es decir, le resultó efectivo el razonamiento lógico matemático que se hizo en el video.

Ahora bien, en la segunda pregunta realizada a cada uno de los participantes, para el Participante 1,2, $3\left(\mathrm{P}_{1,2,3}\right)$, los 3 coinciden en cuanto a la pregunta realizada por el entrevistador, en que la narración fue lo bastante asertiva, puesto que, todos afirman positivamente, por lo que se puede evidenciar que a los tres participantes les gustó la narración.

La última pregunta realizada va dirigida más a sugerencias y recomendaciones, con el fin último de indagar un poco más sobre en como ellos procesan aún más la información y así aplicárselos al video posteriormente, por lo que, para el primer participante este dice que se le debe agregar algo de música, por lo que se puede inferenciar someramente que puede procesar la información de manera más auditiva, entre tanto, para la segunda participante, ésta no recomendó mejorar nada, por lo que, se puede evidenciar que procesa la información tanto de forma auditiva y visual, siendo estas muy abstractas, es decir, que su aprendizaje es de ambos siempre y cuando la información no este sobre cargada. Para el tercer participante, este señala que se le debe colocar música y que los objetos aparezcan uno a la vez, conceptualizando así que posee un aprendizaje tanto de audio y visual al recomendar colocar algo de música y que las animaciones al final del video aparezcan una a la vez, es decir, procesa la información de las dos formas.

Por lo tanto, según los resultados obtenidos en esta última parte, se debe tomar en cuenta las recomendaciones realizadas por cada uno de los informantes para así mejorar el video en cuanto a diseño del mismo.

\section{CONCLUSIONES}

A continuación se describen cada uno de los alcance finales que tuvo este estudio en el cumplimiento de sus objetivos:

Fue propuesto el diseño instruccional empleando tecnología de información y comunicación (TIC) la cual optimizó el proceso de aprendizaje en un tópico de Química General en la que los estudiantes en estudio tenían dificultad para su compresión.

A través de la realización del estudio de necesidades se logró conocer el tópico de Química General y la TIC emergente, resultando ser estas el tema de Equilibrio 
Químico y que gracias a las entrevistas seguidamente realizadas a los participantes en estudio se pudo delimitar aún más el problema, determinando que en dicho tema el Equilibrio Químico Gaseoso era donde se encontraba la problemática sobre todo en los análisis de la constante de equilibrio y predicción de la reacción y para la estrategia instruccional señalaron Video Educativo, como medio audiovisual para el reforzamiento del aprendizaje en los estudiantes del curso de Química General corte 2016-2.

El Diseño a la estrategia didáctica resultante fue la realización de un video educativo correspondiente al tópico de Equilibrio Químico en fase homogénea, por ende, la consulta de fuentes como proyectos desde los tipo documentales hasta factibles en el ámbito de las tecnologías en pedagogía fueron de gran relevancia en la realización del producto surgido como Video Educativo, en los permitiendo así optimizar el proceso de aprendizaje en los estudiantes del curso de Química General.

La validación de la estrategia didáctica surgida bajo enfoque TIC por juicio de expertos (3 docentes) permitió realizar ajustes posteriores en cuanto a contenido, diseño, y estructura y nivel de profundidad de los contenidos a abordar, que permitieron resaltar la calidad de la propuesta.

Una vez aplicado el Video Educativo, se pudo observar que mejoró notablemente el proceso de aprendizaje de los participantes en estudio, debido a que por medio de las entrevistas posteriormente aplicada la estrategia tecnológica estos especificaron que el video educativo estaba bien explicado, los ejercicios resueltos estaban bien razonados y que en aspectos generales estaba bien hecho, sin embargo, dieron algunas recomendaciones en cuanto a diseño que serán tomadas en cuenta puesto que se consideran de gran relevancia.

Se determinó cualitativamente que la aplicación de la estrategia Instruccional empleando Tecnologías de Información y Comunicación resultó ser una alternativa idónea cuando se presentan casos en donde los estudiantes se les dificulta entender ciertos aspectos, en este caso, para el tema de Equilibrio Químico en fase gaseosa, la utilización de los videos educativos resultó ser satisfactorio y contribuye un aporte significativo tanto para los beneficiarios como para autor del presente trabajo de investigación.

A continuación, se presentan las recomendaciones que se derivan del análisis e interpretación de los resultados obtenidos en el trabajo de investigación.

1. En primera instancia, se recomienda aplicar el presente estudio a un tamaño considerable de estudiantes, puesto que para el presente trabajo se aplicó a un grupo reducido por la baja matricula del curso.

2. Se recomienda ampliar el estudio de forma cuantitativa para conocer numéricamente la cantidad de estudiantes que requieran aplicar estrategias bajo enfoque tecnológico debido a que la población actual de alumnos en su mayoría manejan dichas tecnologías de forma amplia.

3. A los docentes, se recomienda que utilicen como estrategias didácticas alternativas el uso de las tecnologías puesto que van de la mano con la capacidad actual del estudiante en el uso y manejo de la innovación tecnológica.

4. Se recomienda, al momento de realizar videos educativos o instruccionales la utilización de la aplicación Scream Recording, puesto que el mismo permite grabar lo que ocurre en pantalla, siendo Screan Cast o Matica las más sencilla de usar para la elaboración de los mismos, y no ocupa espacio en la RAM del disco duro ya que es un ejecutable 
REFERENCIAS

Arias, F. (2009). Proyecto de investigación. Guía para la elaboración. Caracas: Episteme

Bergmann, J., y Sams, A. (2016). Sala de aula invertida: uma metodologia ativa de aprendizagem. Rio de Janeiro: LTC

Busquets, T., Silva, M., y Larrosa, P. (2016). Reflexiones sobre el aprendizaje de las ciencias naturales: Nuevas aproximaciones y desafíos. Estudios pedagógicos (Valdivia), 42(ESPECIAL), 117-135

Castillo, E. y Martínez, N. (2011). Diseño de un taller de capacitación para la elaboración de videos educativos con la herramienta Windows movie maker. Trabajo de grado. Caracas: UPEL-IPC

Diez de Tancredi, D. (2001). Una propuesta metodológica para la producción de materiales educativos. Revista de Investigación, 48, 11-37

EducarChile (2008). Metodología indagatoria para aprender ciencia. [Documento en línea] Disponible: http://www.educarchile.cl/Portal.Base/ Web/VerContenido.aspx?ID=186632. [Consulta: 2018, septiembre 04]

EDUCREA, (2018). Las TIC en el ámbito educativo. [Documento en línea]. Disponible: https://educrea.cl/las-ticsen-el-ambito-educativo/ [Consulta: 2018, octubre 07]

Gardié, O. (1995). Modelo de Enseñanza creativa para la formación y desempeño del docente venezolano. Universidad Pedagógica Experimental Libertador. Tesis doctoral
Hernández, R., Fernández, C., y Baptista, P. (2006). Metodología de la investigación (Vol. 4). México

Kofman, H. (2005). Nuevos contenidos y metodologías con TIC en la Enseñanza de la Física. Revista de Física de la Argentina (FCEFyN UNC) Número extraordinario. Pp. 20-27

Not, L. (1987). Las pedagogías del conocimiento, El profesor ante la integración escolar: Investigación y Formación. Argentina: Editorial Cincel

Pérez, H. (2010). Uso de las tecnologías en el proceso de Enseñanza-Aprendizaje. Semanario: Universidad

Sabino, C. (2002). El proceso de investigación. $\left(3^{\circ} \mathrm{Ed}\right.$.). Caracas: Panapo.

Strauss, A. L., Corbin, J., y Zimmerman, E. (2002). Bases de la investigación cualitativa: técnicas y procedimientos para desarrollar la teoría fundamentada.

Szczurek, M. (1990). Tecnología educativa y Tecnología Instruccional. Revista de tecnología educativa. 4, 257-254

Szczurek, M. (1990). Tendencias actuales de la Tecnología Educativa. Ponencia presentada en las Cuartas Jornadas de Tecnología Educativa en Venezuela, Caracas

Szczurek, M. (1989). La estrategia instruccional. Revista de Investigación y Postgrado, 4(2)

Tuyú Tecnology, (2017). Las TIC en la sociedad actual. [Página web en línea]. Disponible: https://www.tuyu.es/las-ticen-la-sociedad-actual/ [Consulta: 2018, enero 21] 